(1) CrossMark

Cite this: Soft Matter, 2014, 10, 7103

Received 9th April 2014

Accepted 1st July 2014

DOI: $10.1039 / \mathrm{c} 4 \mathrm{sm} 00774 \mathrm{c}$

www.rsc.org/softmatter

\title{
Z-cone model for the energy of an ordered foam
}

\author{
Stefan Hutzler, ${ }^{*}$ Robert P. Murtagh, David Whyte, Steven T. Tobin† and Denis Weaire \\ We develop the Z-Cone Model, in terms of which the energy of a foam may be estimated. It is \\ directly applicable to an ordered structure in which every bubble has $Z$ identical neighbours. The energy \\ (i.e. surface area) may be analytically related to liquid fraction. It has the correct asymptotic form in \\ the limits of dry and wet foam, with prefactors dependent on Z. In particular, the variation of energy with \\ deformation in the wet limit is consistent with the anomalous behaviour found by Morse and \\ Witten [Europhysics Letters, 1993, 22, 549] and Lacasse et al. [Physical Review E, 54, 5436], with a \\ prefactor $Z / 2$.
}

\section{Introduction}

Many of the physical properties of foams may be understood in terms of the minimisation of surface area, under appropriate constraints. This is the condition for static equilibrium, since total energy is proportional to surface area if gas and liquid are treated as incompressible.

Brakke's Surface Evolver ${ }^{1}$ provides a practical method to compute such equilibrium structures, represented by finely tessellated surfaces. It is natural to seek simpler representations and models to provide estimates of energies and forces, even at the expense of drastic approximations, such as pairwise interaction potentials between bubbles. ${ }^{2,3}$

This has raised a number of questions, addressed by Morse and Witten ${ }^{4}$ and Lacasse et al. ${ }^{5}$ How valid is the assumption of pairwise additive potentials? What is the true form of interaction (i.e. the change in surface area) between two bubbles which barely touch each other? We offer a new approximate formulation, the $Z$-Cone Model, that advances our understanding of such questions, in terms of analytic solutions of the model.

As in the work of Lacasse et al., we consider a foam in which each bubble has $Z$ equivalent neighbours, for example the facecentred cubic structure $(Z=12)$. We seek to evaluate the energy (or surface area) as a function of $Z$ and the degree to which the bubbles are compressed together (that is, the liquid fraction). Both gas and liquid are treated as incompressible.

Our essential geometrical approximation is inspired by Ziman's early description of the Fermi surface of copper. ${ }^{6}$ The bubble volume can be divided into $Z$ equivalent objects which meet at a central point. We take one such object and approximate it by a cylindrically symmetric cone of the same solid angle and volume, as shown in Fig. 1. Note that these new cones, if assembled, would 'overlap' since cones cannot tile 3D space.

School of Physics, Trinity College Dublin, Dublin, Ireland.E-mail: stefan.hutzler@tcd.ie $\dagger$ Present address: University of Melbourne, Melbourne, Australia.
The equivalence of the $Z$ sections corresponds to a regular polyhedron in the dry limit; such regular polyhedra have been the starting point for theories of dry foams. ${ }^{7}$
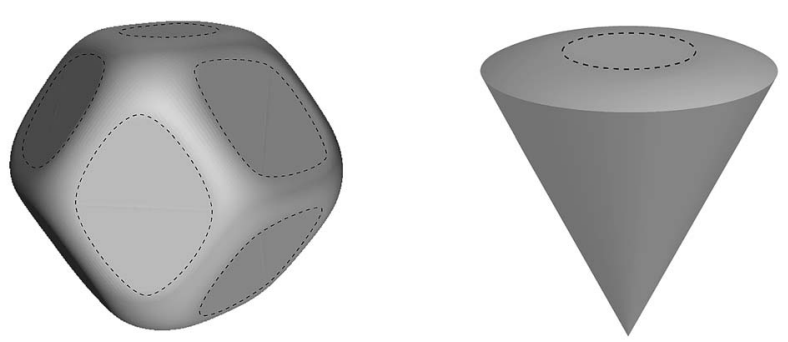

(b)

(a)

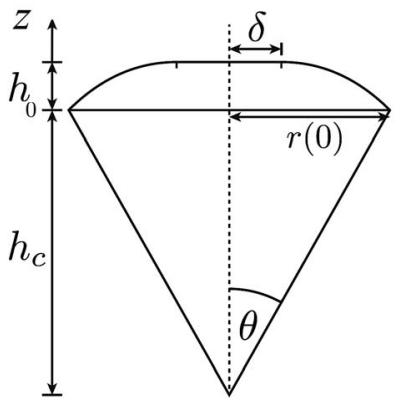

(c)

Fig. 1 The shape of a bubble in a crystalline foam with $Z$ equivalent neighbours, shown in (a) for $Z=12$, may be approximated by an assembly of $Z$ cones of the type shown in (b). Its flattened surface corresponds to a bubble-bubble contact. (c) 2D cross-section of a cone with relevant notation. During bubble deformation, total bubble volume $V$ and total solid angle must be conserved, according to $V=Z V_{\mathrm{c}}$ and $4 \pi=Z \Omega$, where $V_{\mathrm{c}}=\frac{2}{3} \pi R_{0}{ }^{3}(1-\cos \theta)$ is the volume of a cone with opening angle $\theta=\arccos (1-2 / Z), R_{0}$ is the radius of the spherical sector (corresponding to an undeformed cone) and $\Omega$ is the solid angle of the cone. 
A cone's surface consists of a flat disk of area $\pi \delta^{2}$ (the area of contact with a neighbouring bubble) and an outer part which has constant mean curvature, terminating at a right angle to the cone surface. Flat disk and outer part join smoothly: there is no curvature discontinuity at the boundary.

As the liquid volume fraction $\phi$ is reduced, the contact area grows, and the separation of bubble centres $2\left(h+h_{\mathrm{c}}\right)$ is reduced according to:

$$
2\left(h+h_{\mathrm{c}}\right)=2 R_{0}(1-\xi)
$$

where $h$ and $h_{\mathrm{c}}$ are defined as in Fig. 1(c), $R_{0}$ is the radius of a spherical sector of volume $V_{\mathrm{c}}$ (the volume of a cone) and the deformation, $\xi$, is a dimensionless parameter.

For given $\xi$ and solid angle, we can calculate the bubble surface area $A$ and its total volume $Z V_{\mathrm{c}}$ analytically. For the case of $Z=2$, the cone model is exact and the resulting elliptic integrals have been performed before by Lacasse et al.; ${ }^{5}$ here we generalise their application to other values of $Z$.

Our aim is to compute the dimensionless excess energy $\varepsilon$, defined as:

$$
\varepsilon(\xi)=\frac{A(\xi)}{Z A_{0}}-1
$$

where $A(\xi)$ is the surface area of a bubble as a function of deformation and $A_{0}=2 \pi R_{0}^{2}(1-\cos \theta)$ is the curved surface area of the undeformed (spherical) cap, i.e. for $\xi=0$.

We find that the results of Surface Evolver simulations are well described by the analytic predictions for $\varepsilon(\xi)$ obtained from the cone model. For low deformation, we find a logarithmic dependence on deformation, similar to Lacasse et $a .^{5}$ and Morse and Witten. ${ }^{4}$

Furthermore, we show equivalent calculations for the variation of energy and osmotic pressure with liquid fraction, and discuss some possible extensions of the model.

\section{Mathematical framework}

Our derivation follows that of Lacasse et $a .^{5}$ (the EulerLagrange formalism under the constraint of constant bubble volume) but requires a different boundary condition where the curved part of the surface meets the cone, i.e. $\left.\frac{\mathrm{d} r}{\mathrm{~d} z}\right|_{z=0}=\cot \theta$, where $r(z)$ is the radius of the surface of revolution at $z$ : see Fig. 1(c). The second boundary condition is given by $\left.\frac{\mathrm{d} r}{\mathrm{~d} z}\right|_{z=h}=\infty$. This represents a zero contact angle where the cone is terminated by a flat disk, corresponding to a contact with a neighbouring bubble.

A somewhat lengthy derivation results in the following exact expression for the excess energy $\varepsilon\left(\rho_{\delta}, Z\right)$ as a function of $\rho_{\delta}=\delta / r(0)$ :

$$
\varepsilon\left(\rho_{\delta}, Z\right)=\frac{\rho_{\delta}^{2}+\frac{Z}{\sqrt{Z-1}}\left(1-\rho_{\delta}^{2}\right) K\left(\rho_{\delta}, Z\right)}{Z^{-1 / 3}\left(\frac{Z-2}{\sqrt{Z-1}}+6 J\left(\rho_{\delta}, Z\right)\right)^{2 / 3}}-1 .
$$

The deformation $\xi\left(\rho_{\delta}, Z\right)$ is expressed as

$\xi\left(\rho_{\delta}, Z\right)=1-\left[\frac{4 / Z}{\frac{Z-2}{2 \sqrt{Z-1}}+3 J\left(\rho_{\delta}, Z\right)}\right]^{1 / 3} \times\left(\frac{Z-2}{2 \sqrt{Z-1}}+I\left(\rho_{\delta}, Z\right)\right)$.

The functions $I\left(\rho_{\delta}, \theta\right), J\left(\rho_{\delta}, \theta\right)$ and $K\left(\rho_{\delta}, \theta\right)$ are definite elliptic integrals given by:

$$
\begin{aligned}
& I\left(\rho_{\delta}, Z\right)=\int_{\rho_{\delta}}^{1}\left(\rho^{2}-\rho_{\delta}^{2}\right) f\left(\rho, \rho_{\delta}, Z\right) \mathrm{d} \rho \\
& J\left(\rho_{\delta}, Z\right)=\int_{\rho_{\delta}}^{1} \rho^{2}\left(\rho^{2}-\rho_{\delta}^{2}\right) f\left(\rho, \rho_{\delta}, Z\right) \mathrm{d} \rho \\
& K\left(\rho_{\delta}, Z\right)=\int_{\rho_{\delta}}^{1} \rho^{2} f\left(\rho, \rho_{\delta}, Z\right) \mathrm{d} \rho
\end{aligned}
$$

with

$$
f\left(\rho, \quad \rho_{\delta}, Z\right)=\left[\rho^{2} \frac{Z^{2}}{4(Z-1)}\left(1-\rho_{\delta}^{2}\right)^{2}-\left(\rho^{2}-\rho_{\delta}^{2}\right)^{2}\right]^{-1 / 2} .
$$

\section{Results}

\subsection{Dependence of energy on deformation and liquid fraction}

In the following, we focus on the comparison of the cone model with Surface Evolver simulations of the face-centred cubic (fcc) structure, which is observed experimentally in wet foams. Our model is directly applicable in this case since each bubble has $Z=12$ equivalent neighbours. In the dry limit, a bubble approaches a rhombic dodecahedron. Later, we also show the results of Surface Evolver simulations for a pentagonal dodecahedron, for which the cone model gives even better agreement.

Fig. 2(a) shows that for $Z=12$, over a limited range of $\xi$, the dependence of $\varepsilon$ on $\xi$ is roughly quadratic, as Lacasse et al. obtained from Surface Evolver calculations. For other values of $Z$, the cone model predicts similar behaviour-again, in line with Surface Evolver calculations.

Looking more closely (see Fig. 2(b)), we see deviations from the quadratic form both at small and large $\xi$, corresponding to the limits of a wet and dry foam. We will therefore examine the asymptotic limits, turning our attention for the moment to the variation of excess energy with liquid fraction: see Fig. 3.

The liquid fraction $\phi$ lies between 0 , the dry limit, and a value $\phi_{\mathrm{c}}$ at which the deformation vanishes (the wet limit). It may be expressed in terms of $\xi$ by

$$
\xi=1-\left(\frac{1-\phi_{\mathrm{c}}}{1-\phi}\right)^{1 / 3}
$$

We can obtain a simple expression for $\phi_{\mathrm{c}}$ for the circular cones of the cone model:

$$
\phi_{\mathrm{c}}=\frac{3-4 / Z}{Z-1} .
$$




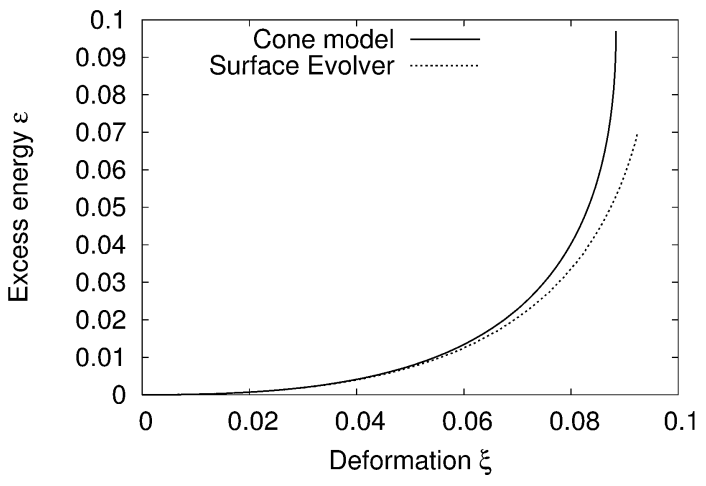

(a)

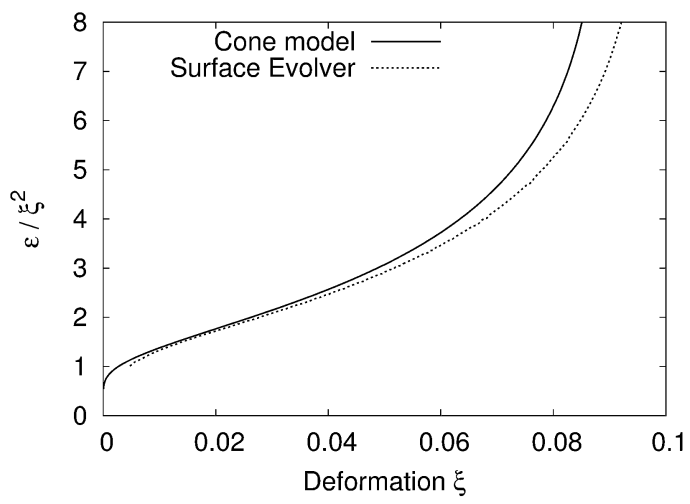

(b)

Fig. 2 (a) Variation of excess energy $\varepsilon$, and (b) variation in $\varepsilon / \xi^{2}$, with deformation $\xi$. The dotted line corresponds to Surface Evolver calculations for the fcc structure shown in Fig. 1(a). (Numerical noise does not allow us to produce reliable Surface Evolver data for $\xi<\sim 0.005$.) The dependence of $\varepsilon$ on $\xi$ may be approximated by a quadratic only over a very limited range of $\xi$.

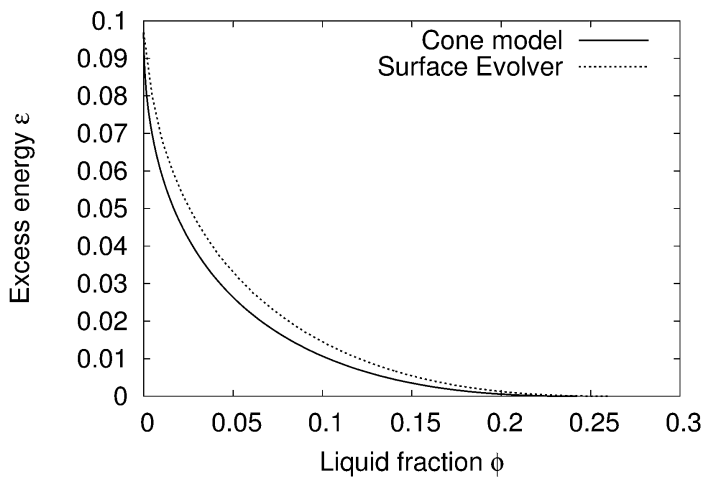

Fig. 3 Variation of energy with liquid fraction: cone model prediction $(Z=12)$ and Surface Evolver calculation for fcc.

Since the cones are only a representation of the actual bubble (see section 1), our relationship between $\xi$ and $\phi$ is hence an approximation.
In the dry limit, $\phi \rightarrow 0$, our cone model data is well described by $\varepsilon(\phi)=e_{0}-e_{1} \phi^{1 / 2}$. This relation may be derived from the decoration of film intersections with Plateau borders of finite cross-section. ${ }^{3}$ The values for the constants $e_{0}$ and $e_{1}$ are close to the true coefficients for the given crystal structure; they vary as $e_{0}=\left[\frac{Z(Z-1)}{(Z-2)^{2}}\right]^{1 / 3}-1$ and $e_{1} \propto 1 / Z$.

In the wet limit, $\phi \rightarrow \phi_{c}$, the energy varies with liquid fraction as

$$
\varepsilon(\phi) \approx-\frac{Z}{18\left(1-\phi_{\mathrm{c}}\right)^{2}} \frac{\left(\phi_{\mathrm{c}}-\phi\right)^{2}}{\ln \left(\phi_{\mathrm{c}}-\phi\right)},
$$

see the discussion in section 3.2.

Fig. 4(a) shows that in the case of a regular pentagonal dodecahedron, the cone model gives an even better prediction for $\varepsilon(\xi)$ than for the fcc arrangement. To further demonstrate the applicability of the cone model, in Fig. 4(b) we show the case $Z=6$ : a bubble confined in a cube.

\subsection{Asymptotic form of energy-deformation relation}

Now turning to the variation of energy with deformation, we note that the wet limit is more subtle. Previous authors ${ }^{4,5}$ have

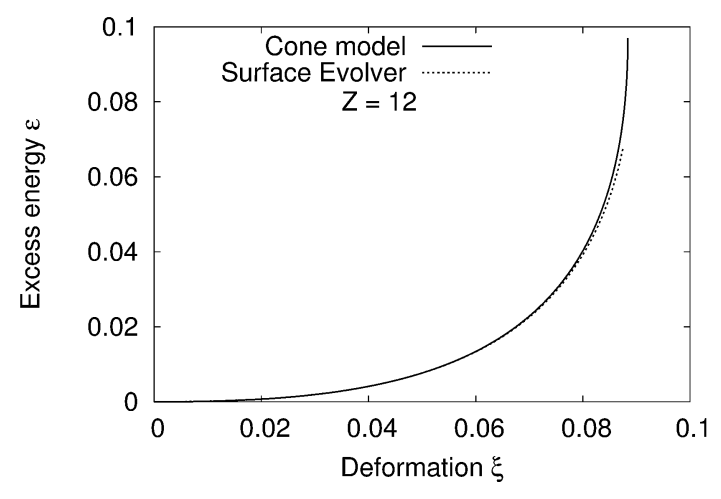

(a)

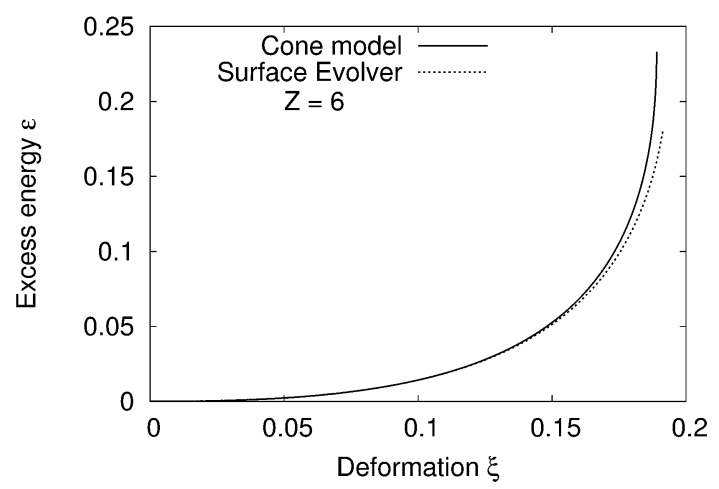

(b)

Fig. 4 Comparison of cone model predictions for $\varepsilon(\xi)$ with Surface Evolver simulations for Platonic solids. (a) $Z=12$ : a pentagonal dodecahedron, and (b) $Z=6$ : a cube. We see good agreement, due to the underlying symmetry of these shapes. Note that for $Z=2$ (not shown) the cone model is exact. 
derived an asymptotic form for the dependence of energy $\varepsilon$ on force $f$ at small deformation, obtaining $\varepsilon \propto f^{2} \ln (1 / f)$. This was derived for the special cases of a droplet pressed against a flat surface $^{4}$ and a droplet compressed by two parallel plates (corresponding to $Z=2$ in our $Z$-cone model). ${ }^{5}$ For present purposes, it is more convenient to consider the energy-deformation relation, which takes the corresponding asymptotic form:

$$
\varepsilon=\mathrm{c} f^{2} \ln (1 / f) \Rightarrow \varepsilon=\frac{\xi^{2}}{4 c \ln \xi},
$$

where $c$ is a constant. This result has not been previously stated: its validity may be checked by differentiating eqn (4), writing $\frac{\mathrm{d} \varepsilon}{\mathrm{d} \xi}=f$, and keeping lowest order terms. The curves calculated for $\varepsilon(\xi)$ using analytic functions, such as that of Fig. 2(a), all show variations close to $\xi=0$ that are consistent with the above form; see also Fig. 5.

Expansion of the integrals involved (eqn (2)) in the limit $\xi \rightarrow 0$, $\rho_{\delta} \rightarrow 0$ reveals a logarithmic singularity of the form

$$
\varepsilon=-\frac{Z \xi^{2}}{2 \ln \xi} \text {. }
$$

Note that this asymptotic form contains the factor $Z$, rather than $Z^{2}$, as in the approximate fit by Lacasse et al. ${ }^{5}$ for higher values of deformation. Expressing $\xi$ in terms of $\phi$ and Taylor expanding to lowest order leads to the energy variation $\varepsilon(\phi)$ as stated above, eqn (3).

An asymptotic expression that covers a larger range of deformations may be obtained by expressing energy $\varepsilon$ in terms of $\rho_{\delta}$, resulting in

$$
\varepsilon\left(\rho_{\delta}, Z\right)=c_{1} \rho_{\delta}^{4}\left(c_{2}-\ln \rho_{\delta}\right),
$$

with:

$$
c_{1}(Z)=\frac{4}{Z}(1-1 / Z)^{2},
$$

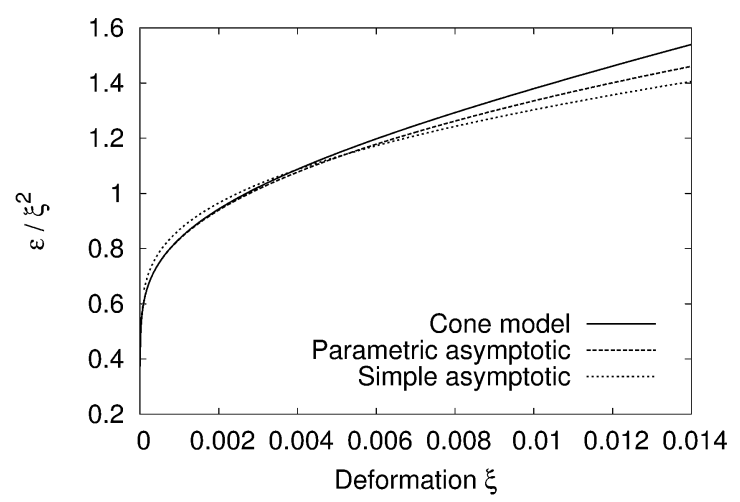

Fig. 5 Asymptotic behaviour of energy $\varepsilon / \xi^{2}$ in the limit of small deformation, $\xi \ll 1$, for $Z=12$. The analytic result for the cone model (solid line) is well represented by a parametric plot of the expansion of eqn (6), together with eqn (1). The dotted line shows the simple expression of eqn (5) which has merit for further investigations of the wet limit, e.g. in the context of osmotic pressure variation. and

$$
c_{2}(Z)=\frac{1}{4}\left(\frac{1}{1-1 / Z}-3\right)-\ln (1-1 / Z) .
$$

Combination with eqn (1) leads to the parametric plot shown in Fig. 5 which describes the analytical result very well for values up to $\xi \approx 0.005$.

The anomalous asymptotic form for the interaction of bubbles as they come into contact appears to be quite general. Only for larger values of $\xi$, and over a limited range, which is a decreasing function of $Z$, may the excess energy be reasonably well approximated as quadratic. The corresponding spring constants increase roughly linearly with $Z$. For values of $Z$ exceeding around 7 this is no longer a good approximation: see Fig. 2(b) for $Z=12$.

\subsection{Osmotic pressure}

This anomalous behaviour also poses a challenge for experiment: can the small forces involved (of the order of $1 \mu \mathrm{N}$ for a single pair of bubbles of radius $1 \mathrm{~cm}$ and $\xi \approx 0.005$ ), or the corresponding variation of osmotic pressure, i.e. the variation of surface energy $E$ with total foam volume $V$ for constant gas volume $V_{\mathrm{g}}, \Pi(\phi)=-\left(\frac{\partial E}{\partial V}\right)_{V_{\mathrm{g}}}$, be readily measured? Here we consider the reduced osmotic pressure, $\tilde{\Pi}=\Pi /(\gamma / R)$, where $\gamma$ is the surface tension of the liquid and $R$ the mean bubble radius. This is a dimensionless quantity which is independent of material properties.

Fig. 6 shows $\tilde{\Pi}(\phi)$, as computed numerically for the cone model, using $\tilde{\Pi}(\phi)=-3(1-\phi)^{2} \frac{\partial \varepsilon}{\partial \phi}$. Using eqn (5), this results in

$$
\tilde{\Pi}=-\frac{Z}{3} \frac{(1-\phi)^{2}}{\left(1-\phi_{\mathrm{c}}\right)^{2}} \frac{\left(\phi_{\mathrm{c}}-\phi\right)}{\ln \left(\phi_{\mathrm{c}}-\phi\right)}
$$

in the wet limit, again also in good agreement with Surface Evolver data with the appropriate choice for $\phi_{\mathrm{c}}$. We further note

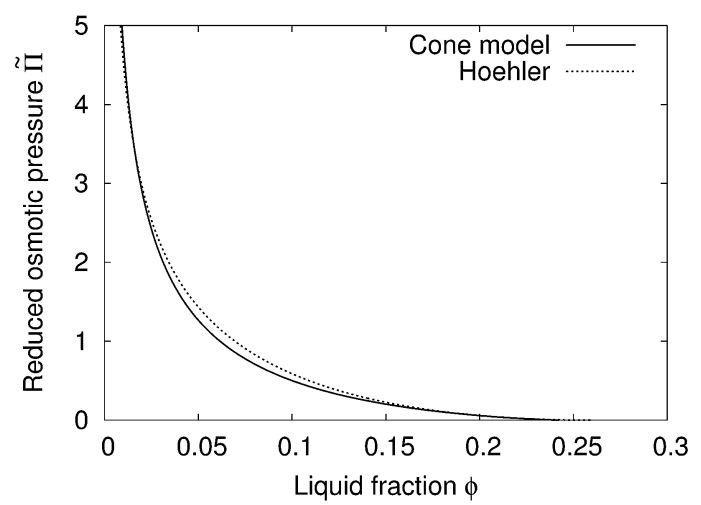

Fig. 6 The variation of the reduced osmotic pressure $\tilde{\Pi}=\Pi /(\gamma / R)$ as a function of liquid fraction, together with an empirical relationship proposed by Höhler et al. to describe experimental data ${ }^{8}$ for ordered foams $(Z=12)$. 
that the cone model gives a good approximation of experimental data for osmotic pressure measurements on fcc-ordered foams ${ }^{8}$ which were summarised in the empirical relationship

$$
\tilde{\Pi}(\phi)=7.3 \frac{\left(\phi-\phi_{\mathrm{c}}\right)^{2}}{\sqrt{\phi}} .
$$

The variation of $\Pi(\phi)$ may also be used to derive a liquid fraction profile under gravity. ${ }^{3,9}$

\section{Cone model applied to a Kelvin foam}

A foam consisting of equivalent cells with $Z$ neighbours cannot be realised except in very few special cases (already stated), not including, for example, bcc (the Kelvin foam). Take the case of $Z=6$ (as used in Fig. 4), for which a simple cubic cell is appropriate, except that it is unstable according to the rules of Plateau: in the dry limit it has $90^{\circ}$ angles at each edge, instead of the required $120^{\circ}$. Nevertheless the estimates which arise from the $Z$-cone model has proven useful in a broader context. Note that the flat-sided cube which is represented in the dry limit is not the same thing as the "isotropic Plateau polyhedron" used by Hilgenfeldt et $a .^{7}$ and Evans et al. ${ }^{10}$ The latter represents a single cell in an extended foam, so that it may be given the appropriate Plateau geometry for stability.

Since the Kelvin foam plays a central role in many analyses it is worthy of some comment here. The dry foam has 14 contacts for each bubble but these consist of eight [111] contacts and six [100] contacts. As liquid fraction is increased, the [100] contacts are lost, provoking instability. ${ }^{11}$ For purposes of calculation with the Surface Evolver, the instability may be suppressed.

If we consider the two cases $Z=8$ and $Z=14$ for the $Z$-cone model, the two curves for $\varepsilon(\phi)$ are found to bound the Surface Evolver result above and below; see Fig. 7. If $Z$ is regarded as a fitting parameter, there is a good correspondence for $Z \approx 10.5$.

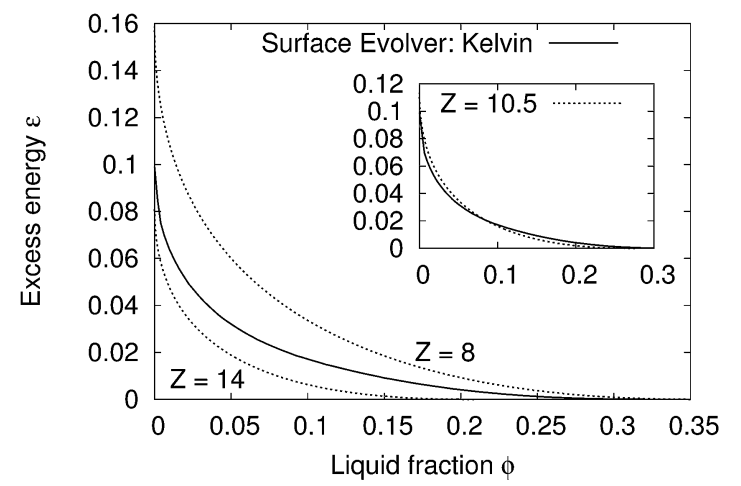

Fig. 7 The variation of excess energy with liquid fraction for a Kelvin cell, as computed with Surface Evolver (solid line). At low $\phi$, this lies close to the cone model curve for $Z=14$, and for higher $\phi$ it lies closer to the $Z=8$ curve. The inset shows reasonable agreement with the cone model data for an effective value of $Z=10.5$.

\section{Outlook}

The asymptotic variation of energy and forces in the wet limit is of some topical importance, because a wet foam is regarded as an ideal experimental system with which to investigate jamming properties, ${ }^{\mathbf{1 2}}$ since it has well-characterised constituents without static friction. ${ }^{\mathbf{1 3}}$ Theories of jamming often invoke the kind of quadratic or Hertzian forces ${ }^{\mathbf{1 4 , 1 5}}$ that we now know to be inappropriate for foams, in the wet limit.,

In this way, the cone model ties together a number of previous results with a single coherent picture, with the correct asymptotic forms, based entirely on analytic expressions.

The model is strictly only applicable to a limited number of cases, in which neighbours are equivalent. However, it should be possible to pursue its generalisation, so that $\varepsilon(\phi)$ can be predicted for cases such as that of a typical random foam, in which $Z$ varies with $\phi$, similarly to how the model of strictly regular isotropic Plateau polyhedra can give insight into the properties of disordered dry foams. ${ }^{10}$ It will, for example, be necessary to take into account the role of near neighbours in determining the Voronoi cell size and hence $\phi$. As a first step, we are in the process of extending the cone model to ordered bidisperse foams with curved bubble-bubble contact areas.

The division of the bubble into cones is reminiscent of the granocentric model used to study random packings, ${ }^{16}$ where spherical particles are placed in cones around a central particle.

So far as we are aware, no experimental test of the logarithmic singularity has been forthcoming. One way of doing so would be to bring two bubbles into contact by increasing their internal pressure, as in the technique employed by Langevin and others ${ }^{17}$ to examine the various stages of film thinning. One might use such apparatus to measure film area as function of thickness, from which the force may be determined.

\section{Acknowledgements}

We wish to thank Siddartha Sen for assisting us in analysing the energy-deformation relation in the wet limit and Thomas A. Witten for helpful correspondence. We also wish to acknowledge COST action MP1106: Smart and Green Interfaces. We thank the anonymous referees for their help in clarifying the manuscript. This work was co-funded by the European Regional Development Fund and the HEA, Ireland.

\section{References}

1 K. A. Brakke, Exp. Math., 1992, 1, 141-165.

2 D. Durian, Phys. Rev. Lett., 1995, 75, 4780-4783.

3 D. Weaire and S. Hutzler, The Physics of Foams, Oxford University Press, Oxford, 1999.

4 D. Morse and T. Witten, Europhys. Lett., 1993, 22, 549.

5 M. D. Lacasse, G. S. Grest and D. Levine, Phys. Rev. E: Stat. Phys., Plasmas, Fluids, Relat. Interdiscip. Top., 1996, 54, 5436-5446.

6 J. M. Ziman, Philos. Mag., 1961, 6, 1013-1034.

7 S. Hilgenfeldt, A. Kraynik, D. Reinelt and J. Sullivan, Europhys. Lett., 2004, 67, 484. 
8 R. Höhler, Y. Y. C. Sang, E. Lorenceau and S. Cohen-Addad, Langmuir, 2008, 24, 418-425.

9 H. Princen, Langmuir, 1986, 2, 519-524.

10 M. E. Evans, J. Zirkelbach, G. E. Schröder-Turk, A. M. Kraynik and K. Mecke, Phys. Rev. E: Stat., Nonlinear, Soft Matter Phys., 2012, 85, 061401.

11 D. Weaire, N. Pittet, S. Hutzler and D. Pardal, Phys. Rev. Lett., 1993, 71, 2670.

12 Jamming and Rheology - Constrained Dynamics on Microscopic and Macroscopic Scales, ed. A. Liu and S. Nagel, Taylor and Francis, London and New York, 2001.
13 M. van Hecke, J. Phys.: Condens. Matter, 2010, 22, 033101.

14 A. J. Liu, S. R. Nagel, W. Van Saarloos and M. Wyart, Dynamical heterogeneities in glasses, colloids, and granular media, Oxford Science Publications, ch. 9, 2011.

15 M. Van Hecke, J. Phys.: Condens. Matter, 2010, 22, 033101.

16 M. Clusel, E. I. Corwin, A. O. Siemens and J. Bruji\&cacute, Nature, 2009, 460, 611-615.

17 A. Sonin, A. Bonfillon and D. Langevin, J. Colloid Interface Sci., 1994, 162, 323-330. 